\title{
Análise da imunoexpressão do PCNA e p53 em carcinoma de células escamosas oral. Correlação com a gradação histológica de malignidade e características clínicas ${ }^{1}$
}

\author{
Adriana Terezinha Neves Novellino ${ }^{2}$ \\ Rivadávio Fernandes Batista de Amorim ${ }^{3}$ \\ Lélia Maria Guedes Queiroz ${ }^{4}$ \\ Roseana de Almeida Freitas ${ }^{5}$
}

\begin{abstract}
$\overline{\text { Novellino ATN, Amorim RFB, Queiroz LMG, Freitas RA. Análise da imunoexpressão do PCNA e }}$ p53 em carcinoma de células escamosas oral: correlação com a gradação histológica de malignidade e características clínicas. Acta Cir Bras [serial online] 2003 Set-Out;18(5). Disponível em URL: http://www.scielo.br/acb.
\end{abstract}

RESUMO - Objetivo: Analisar a imunoexpressão das proteínas PCNA e p53 em carcinoma de células escamosas oral (CCEO) vislumbrando verificar uma possível correlação entre o escore de malignidade e os dados clínicos dos pacientes. Métodos: Selecionou-se 14 casos de CCEO e classificando-os de acordo com uma adaptação do sistema de gradação histológica de malignidade proposto por Anneroth, Batsakis, Luna, enquadrando-se 7 casos como baixo escore de malignidade (Grupo I) e 7 casos como alto escore (Grupo II). As informações dos pacientes foram coletadas através de fichas clínicas e os dados tabulados. Realizou-se, então, o estudo imuno-histoquímico utilizando anticorpos monoclonais anti-PCNA e anti-p53, obtendo-se o índice de positividade (IP) em cada caso. Resultados: Identificou-se um IP médio ao PCNA de 56,0\% no Grupo I e 83,8\% no Grupo II. Com relação à p53 o IP médio no Grupo I e no Grupo II correspondeu, respectivamente, a $41,2 \%$ e 41,4\%. A análise estatística demonstrou correlação significativa entre o índice de células PCNA positivas e o escore de malignidade. Nenhuma outra correlação foi observada. Conclusões: O maior número de células PCNA positivas correlacionou-se com elevado escore de malignidade em CCEOs, sugerindo maior atividade proliferativa nestas lesões, entretanto, tal correlação não se estabeleceu quando da análise da imunoexpressão da p53.

DESCRITORES - Carcinoma de células escamosas. PCNA. Genes p53. Imunohistoquímica. Neoplasias bucais.

\section{Introdução}

Dentre todas neoplasias malignas com sede no complexo maxilofacial, o carcinoma de células escamosas oral (CCEO) é de fato o de maior importância por representar cerca de 90 a $95 \%$ destas, além de estar associado, na maioria das vezes, com altos índices de morbidade e mortalidade ${ }^{1,2}$.
Ao longo dos últimos anos, a avaliação do comportamento biológico de diversas afecções e do fenótipo maligno de várias neoplasias têm sido investigados por uma grande gama de técnicas na área médica e odontológica, a saber: contagem de figuras de mitose, avaliação das regiões organizadoras nucleolares (AgNORs), incorporação de timidina tritiada e autoradiografia, 5-bromodeoxiuridina (Brdu), citometria de

1. Trabalho realizado no Laboratório de Imuno-histoquímica do Programa de Pós-Graduação em Patologia Oral do Departamento de Odontologia (DOD) da Universidade Federal do Rio Grande do Norte (UFRN).

2. Mestre em Patologia Oral, Professora da Disciplina de Patologia Oral da Universidade Gama Filho do Rio de Janeiro (UGF).

3. Doutorando pelo Programa de Pós-Graduação em Patologia Oral, Departamento de Odontologia da UFRN.

4. Professora Doutora, Adjunta do Programa de Pós-Graduação em Patologia Oral, Departamento de Odontologia da UFRN.

5. Professora Doutora, Adjunta e Coordenadora do Programa de Pós-Graduação em Patologia Oral, Departamento de Odontologia da UFRN. 
fluxo, além de novas técnicas em genética e biologia molecular ${ }^{3-6}$. Atualmente, os métodos imuno-histoquímicos são amplamente utilizados neste sentido, sendo o antígeno nuclear de células proliferantes (PCNA), $\mathrm{Ki}-67$ e p53 os mais estudados ${ }^{7,8}$.

O PCNA representa uma proteína nuclear não histônica, que atua como um co-fator da DNA polimerase delta, sendo observada sua presença no ciclo celular na fase G1 tardia, com um pico na fase S e declínio durante a fase G2 e M. Geralmente, a demonstração da imunoexpressão do PCNA permite verificar a verdadeira fração proliferativa da entidade estudada ${ }^{7-10}$.

O gene p53 constitui um gene supressor de tumor e o produto gênico de sua expressão codifica uma fosfoproteína denominada, igualmente, de $\mathrm{p} 53^{11}$. Considerado como o "guardião do genoma", dentre todo o espectro de genes envolvidos na carcinogênese humana, o gene p53 é um dos de maior importância e o conhecimento de seus mecanismos de ação representa uma etapa fundamental para a compreensão dos aspectos biológicos da oncogênese ${ }^{12}$. A proteína p53 possui várias formas de ação, como a parada do ciclo celular em G1 após um dano à estrutura genômica, indução da apoptose em G2 e, ainda, ativação transcricional de vários outros genes relacionados ao ciclo celular. Valese ressaltar que mutações no gene p53, resultando, conseqüentemente, em um proteína mutada e inativa, são identificadas em cerca de 50 a $70 \%$ de todas as neoplasias humanas. Outrossim, a inativação da função fisiológica da p53 pode acontecer por outros mecanismos, incluindo a conjugação com proteínas virais ou interação com a oncoproteína mdm $2^{13,14}$.
$\mathrm{Na}$ tentativa de determinar a tendência comportamental dos tumores, vários sistemas de gradação baseados nos caracteres histológicos são encontrados na literatura ${ }^{15}$. Com este intuito, Anneroth et al. no ano de 1987 recomendaram um sistema de gradação histológica de malignidade do CCEO fundamentado em 2 parâmetros: população celular tumoral (grau de ceratinização, pleomorfismo nuclear e número de mitoses) e resposta do hospedeiro (padrão de invasão, estágio de invasão e infiltrado linfoplasmocitário) ${ }^{16}$. Desde então, diversos trabalhos foram realizados analisando os diferentes sistemas de gradação histológica e seu valor prognóstico ${ }^{17,18}$.

Diante do que foi exposto, o presente trabalho teve como objetivo realizar um estudo imuno-histoquímico das proteínas PCNA e p53 em CCE no intuito de verificar uma possível correlação entre estes marcadores biológicos e o sistema de gradação histológica preconizado por Anneroth et al. ${ }^{16}$, modificado para este estudo, assim como as variáveis clínicas dos pacientes afetados por esta afecção.

\section{Métodos}

Para a realização do presente estudo, foram selecionados 14 casos de CCEO diagnosticados e pertencentes aos arquivos do Serviço de Anatomia Patológica da Disciplina de Patologia Oral, Departamento de Odontologia, Universidade Federal do Rio Grande do Norte. Foram coletadas as informações constantes nas fichas de solicitação de exame histopatológico referentes aos casos selecionados sendo as variáveis clínicas estudadas: sexo, idade, localização anatômica e cor da pele conforme expressa a Tabela 1.

TABELA 1 - Dados clínicos referentes aos pacientes portadores de CCEO estudados.

\begin{tabular}{ccccc}
\hline Paciente & Sexo & Idade & Localização Anatômica & Cor da Pele \\
\hline 1 & Masc. & 65 & Mucosa jugal & Leucoderma \\
2 & Fem. & 87 & Lábio inferior & Leucoderma \\
3 & Masc. & 59 & Língua & Leucoderma \\
4 & Fem. & 60 & Palato & Leucoderma \\
5 & Masc. & 51 & Palato & Leucoderma \\
6 & Masc. & 49 & Lábio inferior & Leucoderma \\
7 & Fem. & 63 & Língua & Leucoderma \\
8 & Fem. & 61 & Palato & Leucoderma \\
9 & Fem. & 73 & Rebordo maxilar & Leucoderma \\
10 & Fem. & 72 & Assoalho bucal & Melanoderma \\
11 & Masc. & 69 & Língua & Feoderma \\
12 & Masc. & 67 & $*$ & Leucoderma \\
13 & Fem. & 54 & Assoalho bucal & Feoderma \\
14 & Masc. & 22 & Rebordo maxilar & Leucoderma \\
\hline
\end{tabular}

* Não havia informação na ficha clínica 


\section{Análise morfológica}

A amostra selecionada foi submetida a uma análise morfológica pela técnica da hematoxilina e eosina, por meio de microscopia de luz, na qual os espécimes foram classificados por 2 observadores previamente calibrados de acordo com o sistema de gradação histológica preconizado por Anneroth et al. ${ }^{16}$ que objetiva tanto a análise da população celular tumoral, como da resposta do hospedeiro, através dos seguintes parâmetros: grau de ceratinização, pleomorfismo nuclear, número de mitoses, padrão de invasão, estágio de invasão e infiltrado linfoplasmocitário. Em virtude do material selecionado para este estudo ser oriundo de biópsias incisionais, fez-se necessária a não utilização do critério "estágio de invasão", a exemplo dos estudos de Pinto $\mathrm{Jr}^{15}{ }^{15}$ o que resultou em um sistema de gradação baseado em 5 parâmetros, para os quais estabeleceu-se um escore que variava de 1 a 4 conforme recomendação dos autores supracitados. Portanto, o resultado da soma total dos escores estabelecidos, foi dividido por $5 \mathrm{e}$ não por 6 , a fim de se obter o escore médio final referente a cada caso. Os casos avaliados foram divididos em 2 grupos com base no escore médio final: Grupo I, baixo escore, sendo enquadrados neste grupo os casos cujo valor médio foi até 2,5 e, Grupo II, aqueles com valores médios iguais ou maiores que 2,6, segundo preconizam Anneroth et al. ${ }^{18}$.

\section{Estudo imuno-histoquímico}

Foram obtidos cortes histológicos de $3 \mathrm{~mm}$ de espessura a partir de amostras teciduais fixadas em formol a $10 \%$ e emblocadas em parafina. Os espécimes foram processados através da técnica imuno-histoquímica pelo método da Estreptoavidina-Biotina, utilizando anticorpos monoclonais anti-PCNA na diluição de 1:80 (clone PC-10) e anti-p53 na diluição de 1:50 (clone DO-7). Os cortes foram submetidos à recupera- ção antigênica através de tratamento por microondas (700W), realizando-se 3 ciclos de 5 minutos cada em ácido cítrico $10 \mathrm{mM}$ (pH 6.0). Após a incubação com os anticorpos primários e secundários, assim como com o complexo Estreptoavidina-Biotina, procedeu-se a revelação dos cortes utilizando-se solução cromógena de diaminobenzidina a $0,03 \%$ e contra-coloração com hematoxilina de Mayer.

Após a marcação imuno-histoquímica foi realizada uma análise quantitativa da expressão das proteínas PCNA e p53, na qual contou-se o número de células positivas em 1.000 selecionadas aleatoriamente em cada caso estudado. O procedimento de contagem foi realizado por meio de microscópio de luz, regulado para o aumento de imersão, ao qual foi adaptado um retículo de Weibel NGW2 para estereologia a fim de se evitar recontagem de células. Por fim foi obtido um índice de positividade (IP) para cada proteína investigada através da fórmula abaixo descrita:

$$
\mathrm{IP}=\frac{\text { Número de células positivas }}{1.000 \text { células aleatoriamente }} 100
$$

\section{Análise estatística}

A análise estatísticas dos dados foi realizada através dos testes " $t$ " de Student para amostras dependentes e independentes e uma análise descritiva a fim de verificar alguma relação entre a imunoexpressão das proteínas estudadas e o escore médio tumoral. Além desta relação, testou-se, também, a correlação entre estes resultados e os dados clínicos dos pacientes.

\section{Resultados}

\section{Resultados morfológicos}

Os escores correspondentes aos casos analisados encontram-se registrados na Tabela 2.

TABELA 2 - Escores emitidos para os casos de CCEO em relação aos critérios analisados.

\begin{tabular}{ccccccc}
\hline Caso & Ceratinização & $\begin{array}{c}\text { Pleomorfismo } \\
\text { Nuclear }\end{array}$ & $\begin{array}{c}\text { Figuras } \\
\text { de Mitose }\end{array}$ & $\begin{array}{c}\text { Padrão } \\
\text { de Invasão }\end{array}$ & $\begin{array}{c}\text { Infiltrado } \\
\text { Linfoplasmo-citário }\end{array}$ & $\begin{array}{c}\text { Escore } \\
\text { Médio }\end{array}$ \\
\hline 1 & 2 & 2 & 2 & 3 & 1 & $\mathbf{2 , 0}$ \\
2 & 1 & 3 & 3 & 2 & 1 & $\mathbf{2 , 0}$ \\
3 & 1 & 1 & 3 & 3 & 2 & $\mathbf{2 , 0}$ \\
4 & 3 & 1 & 2 & 2 & 1 & $\mathbf{1 , 8}$ \\
5 & 1 & 1 & 4 & 2 & 2 & $\mathbf{2 , 0}$ \\
6 & 2 & 2 & 1 & 2 & 2 & $\mathbf{2 , 0}$ \\
7 & 2 & 1 & 4 & 2 & 1 & $\mathbf{2 , 6}$ \\
8 & 2 & 3 & 4 & 3 & 3 & $\mathbf{2 , 6}$ \\
9 & 1 & 3 & 4 & 2 & 1 & $\mathbf{2 , 0}$ \\
10 & 4 & 2 & 4 & 2 & 1 & $\mathbf{3 , 2}$ \\
11 & 4 & 3 & 4 & 2 & 3 & $\mathbf{3 , 0}$ \\
12 & 4 & 3 & 4 & 2 & 2 & $\mathbf{3 , 0}$ \\
13 & 3 & 4 & 1 & 4 & & \\
14 & 4 & 4 & & & & \\
\hline
\end{tabular}




\section{Resultados imuno-histoquímicos}

Foram consideradas positivas, as células que apresentavam reação restrita ao núcleo, representada por coloração de cor acastanhada e/ou amarelada, independente da intensidade da coloração. Com relação ao PCNA, todos os casos mostraram-se imunopo-

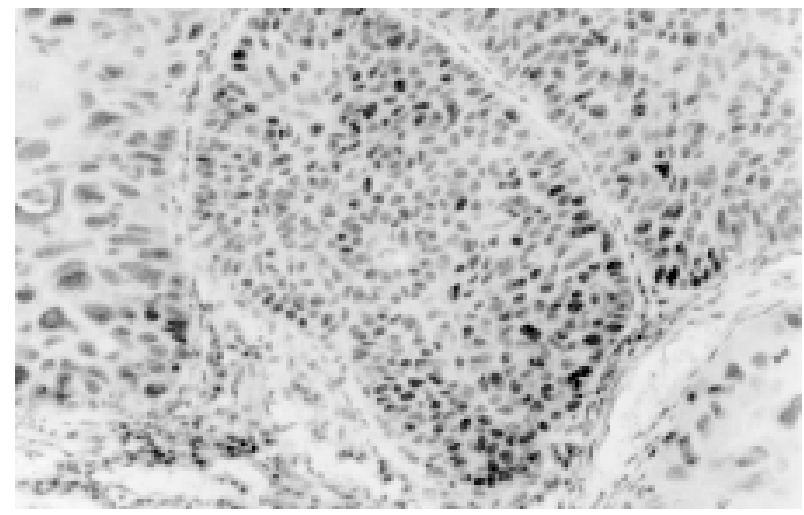

FIGURA 1 - CCEO de alto escore de malignidade. Elevado índice de imunorreatividade ao PCNA (SABC-200x). sitivos, com os IPs variando de $17,0 \%$ a $97,5 \%$ e média total de 69,8\% (Figuras 1 e 2). Já com relação à p53, dos 14 casos investigados, 11 foram imunorreativos com IP variando de 27,0 a $81,7 \%$ e média total de $41,3 \%$ (Figura 3) (Tabela 3).

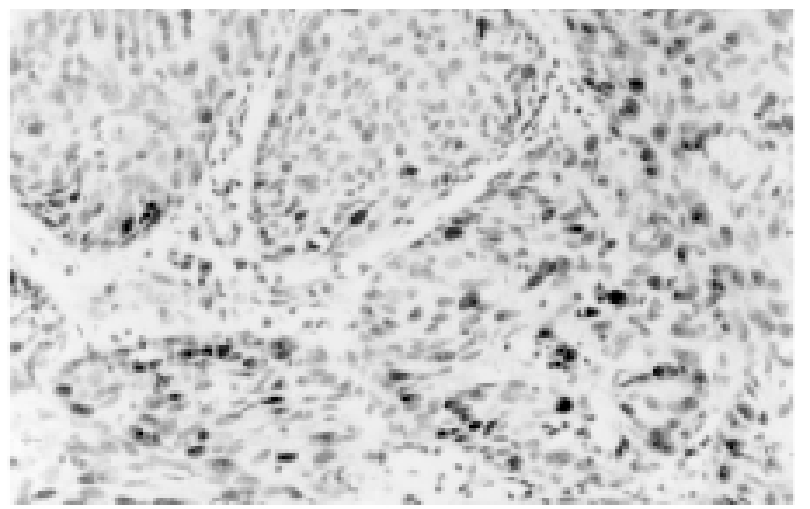

FIGURA 2 - CCEO de baixo escore de malignidade. Reduzido número de células PCNA positivas (SABC-200x).

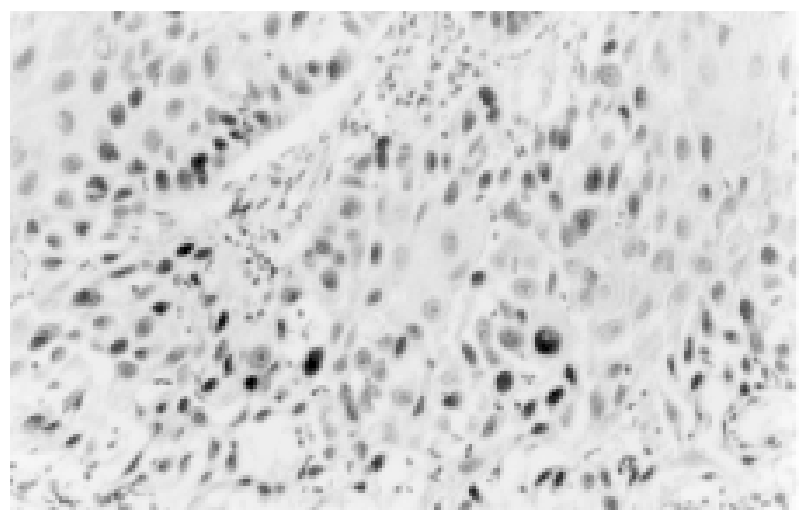

FIGURA 3 - Padrão de imunopositividade à p53 observado nos dois grupos de CCEO (SABC-400x).

TABELA 3 - Número de células PCNA e p53 positivas expresso pelo IP em CCEO de baixo e alto escore de malignidade.

\begin{tabular}{c|cccc}
\hline \multirow{2}{*}{ Caso } & \multicolumn{2}{|c}{ Índice de positividade ao PCNA (\%) } & \multicolumn{2}{c}{ Índice de positividade à p53 } \\
\cline { 2 - 5 } & $\begin{array}{c}\text { Baixo Escore } \\
\text { (Grupo I) }\end{array}$ & $\begin{array}{c}\text { Alto Escore } \\
\text { (Grupo II) }\end{array}$ & $\begin{array}{c}\text { Baixo Escore } \\
\text { (Grupo I) }\end{array}$ & $\begin{array}{c}\text { Alto Escore } \\
\text { (Grupo II) }\end{array}$ \\
\hline 1 & 41,7 & 87,3 & 33,2 & 36,9 \\
2 & 53,6 & 77,3 & 58,1 & 47,3 \\
3 & 17,0 & 87,3 & 59,9 & 74,0 \\
4 & 58,8 & 85,4 & 81,7 & 65,2 \\
5 & 90,2 & 97,5 & - & - \\
6 & 64,9 & 71,5 & 59,5 & - \\
7 & 65,5 & 80,6 & 41,2 & 41,4 \\
Média & 55,9 & 83,8 & & 41,3 \\
Média Total & & 69,8 & & \\
\hline
\end{tabular}

- Não houve imunorreatividade 


\section{Resultados estatísticos}

A análise estatística demonstrou diferença significativa $(\mathrm{p}<0,05)$ entre o IP médio de células PCNA positivas do grupo de baixo escore $(55,9 \%)$ e alto escore $(83,8 \%)$ de malignidade. Não foi observada diferença estatística entre o IP à p53.

No que diz respeito à análise das variáveis clínicas estudadas (sexo, idade, localização anatômica e cor da pele) não se observou nenhuma correlação entre estas e a gradação histológica assim como com os IPs ao PCNA e p53.

\section{Discussão}

Muito se tem pesquisado no sentido de uma melhor compreensão do comportamento biológico do CCEO, com a finalidade da obtenção de melhores resultados terapêuticos, aumentando, por conseguinte, a sobrevida e levando a um melhor prognóstico dos pacientes afetados. Diversas evidências apontam para o fato de que anormalidades nos genes que regulam a proliferação e morte celular são frequientes em carcinomas de células escamosas da região de cabeça e pescoço e estudos têm demonstrado que tanto estas alterações genéticas e a taxa de proliferação celular tumoral podem estar associadas de maneira significativa com o prognóstico e com a resposta ao tratamento ${ }^{19-22}$.

Dentre os biomarcadores empregados para verificar a cinética celular de inúmeras lesões destaca-se o PCNA e sua imunoexpressão é objeto de diversos estudos ${ }^{3,4,9,10,23-25}$. Em conformidade com os dados citados previamente, pode-se observar neste experimento uma diferença significativa da imunorreatividade do PCNA entre os grupos de baixo escore e alto escore de malignidade, ou seja, uma correlação entre a gradação histológica de malignidade e o índice de células positivas. Em outras palavras, detectou-se que os tumores com escores mais elevados, indicando menor grau de diferenciação, apresentavam número de células PCNA positivas significativamente maior que aqueles de baixo escore. Esta relação inversamente proporcional entre o número de células PCNA reativas e o grau de diferenciação tumoral também foi observada por outros autores $^{24,25}$. Robbins et al. ${ }^{25}$ que constataram um aumento do número de células marcadas pelo PCNA em CCEO menos diferenciados e com grande número de figuras de mitose. Interessantemente, o caso que demonstrou o maior número de células PCNA positivas $(90,2 \%)$ no nosso estudo também apresentou maior escore no parâmetro mitose, fato este que vem, pelo menos em parte, a demonstrar a validade do PCNA em detectar a fração proliferativa tumoral.

Em um estudo recente publicado por Tjalma et al. ${ }^{24}$, foi avaliada a importância de vários fatores biológicos, dentre estes o PCNA, em carcinoma cervicais.
Foram selecionados 111 pacientes portadores de carcinoma cervicais invasivos primários com idade média de 52 anos de idades, com tempo de acompanhamento entre 4 a 227 meses (média 50 meses) e, por fim, sendo as características histopatológicas dos espécimes coletadas de acordo com os critérios da Organização Mundial de Saúde. Tais autores puderam identificar uma forte associação da gradação tumoral, status dos linfonodos e o índice de células PCNA positivas, inferindo a validade do PCNA como indicador prognóstico.

Assim como os informes de Tjalma et al. ${ }^{24}$, os achados resultantes da análise quantitativa das células PCNA positivas no presente estudo, sugerem que os casos de escores mais elevados apresentam maior potencial proliferativo, o que contribui, conseqüentemente, para um comportamento pelo menos localmente mais agressivo neste grupo de lesões.

Com relação a imunoexpressão da p53, 3 dos 14 casos analisados não se mostrou reativo, o que pode ser justificado por diferentes fatores: ausência de síntese da p53; conjugação e degradação da p53 por associação a vírus como, por exemplo, associação com a proteína E6 do papiloma vírus humano (HPV); e estabilização e inativação da p53 pela mdm2 $2^{7,10,13,14}$. De acordo com os relatos de Thomas $^{26}$, a ausência de positividade não exclui a possibilidade de mutação genética e, por um outro lado, conforme infere Robbins ${ }^{27}$, a imunorreatividade à p53 não necessariamente indica mutações no gene $\mathrm{p} 53$.

A crucial importância e funcionalidade da p53 na regulação do ciclo celular está cada vez mais estabelecida na literatura. Os avanços obtidos nas técnicas de biologia molecular e genética têm possibilitado o estudo cada vez mais detalhado do genoma humano, especialmente quando o assunto em questão é o gene p53. Em condições de normalidade, a vida-média da p53 selvagem é de aproximadamente 6 a 20 minutos, entretanto, este tempo pode aumentar para 6 horas ou mais caso a proteína esteja mutada. Estes fatores justificam a fraca ou, até mesmo, ausência de imunoexpressão desta proteína em tecidos normais ao passo em que sua presença, especialmente em altas concentrações, é um fato observado em lesões pré-malignas e neoplasias malignas, denotando envolvimento ímpar da p53 no processo oncogenético, principalmente na sua fase inicial ${ }^{7,8,14,23}$.

Os IPs médios observados entre os grupos de baixo e alto escore de malignidade foram bastante semelhantes nos 2 grupos em relação à p53, não havendo, portanto, diferença significativa entre estes. Resultados semelhantes são demonstrados por outros estudos, como o de Gluckman et al. ${ }^{28}$ que não encontraram associação significativa entre a detecção imuno-histoquímica da p53 e nível de agressividade tumoral. Da mesma 
maneira, Yan et al. ${ }^{29}$ realizaram um estudo em CCEO no qual classificaram a sua amostra baseados em outro sistema de gradação histológica e, igualmente, não observaram correlação da reatividade à $\mathrm{p} 53$. Entretanto, de acordo com a literatura consultada, Araújo et al..$^{30}$ foram os únicos autores capazes de verificar tal correlação em CCEO.

Segundo os informes de Bosari, Viale ${ }^{31}$ anormalidades na proteína p53 não apresentam correlações com variáveis clínico-patológicas do CCEO na região de cabeça e pescoço, inclusive com o estágio e gradação tumoral. A análise dos nossos resultados pela marcação da p53, também, aponta para esta afirmação. GonzalesMoles et al. ${ }^{21}$ investigaram a correlação entre o padrão de expressão da proteína p53 e a sobrevida em pacientes com CCEO. Para tanto, tais autores, por meio de técnica imuno-histoquímica com o anticorpo BP5312, analisaram 78 casos de CCEO dos quais cerca de $57 \%(\mathrm{n}=45)$ expressaram imunopositividade, não sendo encontrada, todavia, nenhuma correlação com o prognóstico dos pacientes. Se analisadas em conjunto todas inferências acima, é coerente levantar a possibilidade de que a expressão aumentada da p53 seja mais indicativa de prognóstico em lesões pré-malignas, apontando aquelas com maior risco de transformação maligna e que esta esteja mais envolvida nos processos de carcinogênese inicial conforme cita a literatura, o que justificaria a expressão não exuberante em neoplasias estabelecidas e, também, a ausência de correlação com o prognóstico tumoral citado em alguns estudos ${ }^{12,21,32}$.

No que concerne as variáveis clínicas avaliadas pelo presente estudo (sexo, idade, localização da lesão e cor da pele), não se identificou nenhuma correlação com os IPs da p53 e PCNA nem tampouco com a gradação histológica. Analisando a expressão da ciclina D1 e da p53 em 56 casos de primários de CCEO, Lam et al. ${ }^{33}$ não identificaram nenhuma relação entre a expressão imuno-histoquímica destas proteínas com a idade e sexo dos pacientes estudados o que corrobora os achados do presente experimento.

\section{Conclusão}

O maior número de células PCNA positivas observado em nosso estudo nos CCEO exibindo escores mais elevados de malignidade só vem a reforçar a expectativa de um comportamento mais agressivo neste grupo de lesões. Entretanto, ao nosso ver, a detecção da fração proliferativa tumoral constitui apenas um dos parâmetros a se considerar quando da análise morfológica do CCEO, não se devendo deixar de ter em mente os outros diversos mecanismos envolvidos nas bases moleculares do processo de oncogênese e que podem estar relacionados à sobrevida e ao prognóstico.

\section{Referências}

1. Scully C. Oral squamous cell carcinoma: from a hypothesis about a virus, to concern about possible sexual transmission. Oral Oncol 2002;38:227-34

2. Gervásio OLAS, Dutra RA, Tartaglia SMA, Vasconcellos WA, Barbosa AA, Aguiar MCF. Oral squamous cell carcinoma: a retrospective study of 740 cases in a brazilian population. Braz Dent J 2001;12:57-61.

3. Hall PA, Coates PJ. Assessment of cell proliferation in pathology: What next? Histopathol 1995;26:105-12.

4. Costa AL, Araújo NS, Pinto DS, Araújo VC. PCNA/AgNOR and $\mathrm{Ki}-67 / \mathrm{AgNOR}$ double staining in oral squamous cell carcinoma. J Oral Pathol Med 1999;28:438-1.

5. Leethanakul C, patel V, Gillespie J, Shillitoe E, Kellman RM, Ensley JF, Limwonsgse V, Emmert-Buck MR, Krizman DB, Gutkind JS. Gene expression profiles in squamous cell carcinomas of the oral cavity: use of capture microdissection for the construction and analyses of stage-specific cDNA libraries. Oral Oncol 2000;36:474-83.

6. Kuropkat C, Venkatesan TK, Caldarelli DD, Panje WR, Hutchinson J, Preisler HD, Coon JS, Werner JA. Abnormalities of molecular regulators of proliferation and apoptosis in carcinoma of the oral cavity and oropharynx. Auris Nasus Larynx 2002;29:165-74

7. Mighell A. PCNA and p53. Oral Oncol Eur J Cancer 1995;31B:403-4.

8. Sittel C, Ruiz S, Volling P, Kvasnicka HM, Jungehulsing M, Eckel HE. Prognostic significance of Ki-67 (MIB1), PCNA and p53 in cancer of the oropharynx and oral cavity. Oral Oncol 1999;35:583-9.

9. Oliveira MDC, Novellino ATN, Freitas RA. Expressão do PCNA em epitélio normal, hiperplásico e atípico de mucosa oral. RPG Rev Pos Grad 2000;7:149-54.

10. Birchall MA, Schock E, Harmon BV, Gobé G. Apoptosis, mitosis, PCNA and bcl-2 in normal, leukoplakic and malignant epithelia of the human oral cavity: prospective, in vivo study. Oral Oncol 1997;33:419-25.

11. Fett-Conte AC, Salles ABCF. A importância do gene p53 na carcinogênese humana. Rev Bras Hematol Hemoter 2002;24:85-9

12. Whyte DA, Broton CE, Shillitoe ED. The unaexplained survival of cells in oral cancer: what is the role of p53? J Oral Pathol Med 2002;31:125-33

13. Moll UM, Schramm LM. p53: An acrobat in tumorigenesis. Crit Rev Oral Biol Med 1998;9,23-37.

14. Cadwell C, Zambetti GP. The effects of wild-type p53 tumor suppressor activity and mutant 53 gain-of-function on cell growth. Gene 2001;277,15-30.

15. Pinto Jr DS. Critérios de avaliação do prognóstico - tipo histológico, marcadores imuno-histoquímicos - em biópsias de carcinoma epidermóide da boca [Tese - Doutorado]. Universidade de São Paulo - Faculdade de Odontologia; 1991

16. Anneroth G, Batsakis J, Luna M. Review of the literature and a recommended system of malignancy grading in oral squamous cell carcinomas. Scand J Dent Res 1987;95:229-47.

17. Bryne M, Koppang HS, Lilleng R, Stene T, Bang G, Dabelsteen E. New malignancy grading is a better prognostic indicator than Broders grading in squamous cell carcinoma. J Oral Pathol Med 1989;18:432-7

18. Anneroth G, Batsakis J, Luna M. Malignancy grading of oral squamous cell carcinoma in the floor of the mouth related to clinical evaluation. Scand J Dent Res 1986;94:347-56. 
19. Unal OF, Ayhan A, Hosal AS. Prognostic value of p53 expression and histopathological parameters in squamous cell carcinoma of oral tongue. J Laryngol Otol 1999;113:446-50.

20. Hedge PU, Brenski AC, Caldarelli DD, Hutchinson J, Panje WR, Wood NB. Tumor angiogenesis and p53 mutations: prognosis in head and neck cancer. Arch Otolaryngol Head Neck Surg 1998;124:80-5

21. Gonzalez-Moles MA, Galindo P, Gutierrez-Fernandez J, Sanchez-Fernandez E, Rodriguez-Archilla A, Ruiz-Avila I, Bravo M. P53 protein expression in oral squamous cell carcinoma. survival analysis. Anticancer Res 2001; 21:2889-94.

22. Kuropkat C, Rudolph P, Frahm SO, Parwasresh R, Werner JA. Proliferation markerki-s11: a prognostic indicator for squamous cell carcinoma of the hypopharynx. Virchows Arch 1999;435:590-5.

23. Liu SC, Klein-Szanto AJP. Markers in normal and leukoplakic epithelia. Oral Oncol 2000;36; 145-51.

24. Tjalma WA, Weyler JJ, Bogers JJ, Pollefliet C, Baay M, Goovaerts GC, Vermorken JB, van Dam PA, van Marck EA, Buytaert PM. The importance of biological factors (bcl-2, bax, p53, PCNA, MI, HPV and angiogenesis) in invasive cervical cancer. Eur J Obstet Gynecol Reproduc Biol 2001;97:223-30.

25. Robbins BA, De La Vera D, Ogata K, Tan EM, Nakamura RM. Immunohistochemical detection of proliferating cell nuclear antigen in solid human malignancies. Arch Pathol Lab Med 1987;111:841-5.

26. Thomas DW. p53 in tumor pathology: Can we trust immunocytochemistry? J Pathol 1992;166:329-30.

27. Robbins P. p53 and breast cancer. Int J Surg Pathol 1996; 4:93-110.

28. Gluckman JL, Pavelic ZP, Welkoborsky HJ, Mann W, Stambrook P, Gleich L, Wilson K, Righi P, Portugal LG, McDonald J, Biddinger P, Steward D, Gartside P. Prognostic indicators for squamous cell carcinoma of the oral cavity: a clinicopathologic correlation. Laringoscope 1997;107:1239-44.

29. Yan JJ, Tzeng CC, Jin YT. Overexpression of p53 in squamous cell carcinoma of buccal mucosa and tongue in Taiwan: An immunohistochemical and clinicopathological study. J Oral Pathol Med 1996;25:55-9.

30. Araújo VC, Loyola AM, Pinto Jr DS, Borra RC, Araújo NS. p53 in biopsies of oral squamous cell carcinoma: a comparative study with a malignancy grading system. Oral Oncol 1997;33:5-9.

31. Bosari S, Viale G. The clinical significance of p53 aberrations in human tumors. Virchows Arch 1995;427:229-41.

32. Amorim RFB. Expressão das proteínas p53, PCNA e Bcl-2 em líquen plano oral [Dissertação-Mestrado]. Universidade Federal do Rio Grande do Norte; 2001.

33. Lam KY, Ng IOL, Yuen APW, Kwong DLW, Wei W. Cyclin D1 expression in oral squamous cell carcinomas: clinicopathological relevance and correlation. J Oral Pathol Med 2000;28:167-72.

Novellino ATN, Amorim RFB, Queiroz LMG, Freitas RA. Immunoexpression analysis of PCNA and p53 in oral squamous cell carcinoma: correlation with histological grading of malignancy and clinical features. Acta Cir Bras [serial online] 2003 Sept-Oct;18(5). Available from URL: http://www.scielo.br/acb.

ABSTRACT - Purpose: Analyze the relationship between PCNA and p53 protein imunoexpression, the histological grading of malignancy and the clinical features of oral squamous cell carcinoma (OSCC). Methods: An immunohistochemical analysis was carried out using the streptavidin-biotin method in 14 cases of OSCC. All cases were classified according to an adaptation of the histological malignancy grading system proposed by Anneroth, Batsakis, Luna. Thus, 7 cases were classified as low score (Group I) and 7 cases as high score (Group II) of malignancy. The clinical data from the patients was obtained through their medical files and the immunohistochemical study was performed using monoclonal antibodies anti-PCNA and anti-p53. Finally, it was obtaineid the labeling index (LI) in each case. Results: The LI for PCNA at Group I was 56.0\% and at Group II 83.8\%. The expression of p53 was similar at the studied groups with a LI of $41.2 \%$ and $41.3 \%$ at Group I and II, respectively. There was statistical correlation in PCNA values and score of malignancy. No significant correlation in the overall clinical data was verified. Conclusion: The high number of PCNA positive cells correlated with high score of malignancy indicates evident proliferative activity in these lesions. However, this correlation was not reached in regard to p53 expression.

KEY WORDS - Oral squamous cell carcinoma. PCNA. Genes, p53. Immunohistochemistry. Mouth neoplasms.

Correspondência:

Profa. Dra. Roseana de Almeida Freitas

Conflito de interesse: nenhum Departamento de Odontologia - Universidade Federal do Rio Grande do Norte

Av. Senador Salgado Filho, 1787

Tel.: 59056-000 Natal - RN - Tel/Fax: (84)215-4138

patologiaoral@patologiaoral.com.br

Data do recebimento: $18 / 07 / 2003$

Data da revisão: $26 / 07 / 2003$

Data da aprovação: 07/08/2003 